resolutions ranging from 1.5 to $2.8 \AA$ with micrometer-sized crystals and nano-gram to micro-gram quantities of protein. The IMISX and IMISXcryo method work with readily available, inexpensive materials and are compatible with high-throughput in situ serial data collection at macromolecular crystallography synchrotron beamlines.

1. Caffrey, M., Cherezov, V. (2009) Nature Protocols. 4:706-731.

2. Caffrey, M. (2015) Acta Cryst. F71, 3-18.

3. Huang, C.-Y. et al. (2015) Acta Cryst. D71, 1238-1256.

4. Huang, C.-Y. et al. (2016) Acta Cryst. D72, 93-112.
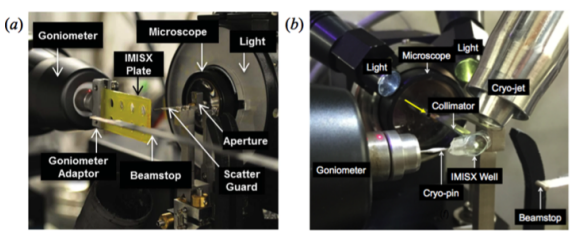

Figure 1. Experimental setup for IMISX data collection at $293 \mathrm{~K}$ (a) and $100 \mathrm{~K}(b)$

Keywords: AlgE, $\beta 2$-adrenergic receptor, bromine SAD, DgkA, experimental phasing, GPCR, in meso, in situ, insulin, lipid cubic phase, mesophase, membrane protein, PepTSt, serial crystallography, sulfur SAD

\section{MS2-P2 Microfluidic devices for fast time-resolved studies}

Diana C.F. Monteiro ${ }^{1}$, Sebastian Bommel ${ }^{1}$, Ramakrishna Vasireddi ${ }^{1}$, Mohammad Vakili ${ }^{1}$, Martin Trebbin ${ }^{1}$

1. Centre for Ultrafast Imaging (CUI) - Universität Hamburg

email: diana.monteiro@uni-hamburg.de

Microfluidics enable the precise control of liquid volumes on the nanoliter scale within micron-sized channels. These very well defined flow conditions make this technology predestined for fundamental time-resolved investigations of biomacromolecules at microfocused X-ray sources. ${ }^{1}$ This approach has many advantages compared to more traditional set-ups in terms of sample consumption, accuracy of measurements and the ability to access time-scales down to $10 \mathrm{~s}$ of $\mu \mathrm{s}$ for time-resolved (TR) studies.

Microfluidic devices can be manufactured using a number of different polymers, such as PDMS, COCs or polyimide films (Kapton ${ }^{\circledR}$ ), each having different optical and mechanical properties and varying ease of manufacture. With the advent of more powerful and brighter X-ray sources, Kapton and COCs have received great interest as materials for X-ray compatible devices due to their low-background and high stability. Alternatively, microfluidic liquid jet devices offer a free-flowing sample stream for XFELs and highly brilliant synchrotron sources. $^{2}$

The precise control of reaction conditions have been demonstrated for the synthesis of nanoparticles ${ }^{3}$ as well as in time-resolved structural studies of biomacromolecules using X-ray scattering ${ }^{1 \mathrm{~b}}$ and diffraction ${ }^{5}$ as well as spectroscopy. ${ }^{6}$ For TR studies, the reaction processes can be initiated by light activation (down to femtoseconds) or rapid mixing, where the time-resolution is only dependent on the diffusion rates of small molecules through the sample thickness $(<1 \mu \mathrm{m}$ in laminar flow conditions, $>10 \mathrm{~s}$ $\mu \mathrm{s})$.

Our group specializes in the design, manufacture and implementation of microfluidic devices, providing sample environments that address specific experimental condition requirements. Alongside this tailored approach, and in collaboration with local synchrotron facilities, we are making standardized microfluidic set-ups for TR biomacromolecular structural studies for general users.

1.(a) Toft, K. N.; et al.; Analytical Chemistry 2008, 80 (10), 3648-3654; (b) Pollack, L. Biopolymers 2011, 95 (8), 543-549.

2.Trebbin, M.; Kruger, K.; DePonte, D.; Roth, S. V.; Chapman, H. N.; Forster, S. Lab on a Chip 2014, 14 (10), 1733-1745.

3.Lazarus, L. L.; et al.; Lab on a Chip 2010, 10 (24), 3377-3379.

4.Rahman, M. T.; et al.; RSC Advances 2013, 3 (9), 2897-2900.

5.Chapman, H. N.; et al.;. Nature 2011, 470 (7332), 73-77.

6.Park, H. Y.; et al.; PNAS 2008, 105 (2), 542-547.

7.Levantino, M.; et al.; Curr Opin Struct Biol 2015, 35 , 41-8.

Keywords: Microfluidics, time-resolved, SAXS 